\title{
Development of Motion Control Camera Design Based on Wires with Anti-sway Method
}

\author{
Taerim Kim, Sungyoung Jung, Gyeongdong Baek and Sungshin Kim* \\ School of Electrical Engineering, Pusan National University, Busan, Korea
}

\begin{abstract}
This paper is proposed about three axis motion control camera design method based on wires. Original motion control camera consists of track, boom, L-Head, Camera and so on and is enormous and expensive. But proposed motion control camera adjusts wire length using encoders and motors. And position control use position based straight line of straight-line move method for moving precise position. Proposed simple design is able to use various place and inexpensive than original motion control camera. But, camera was vibrated and rotated due to basic property of wire. So we proposed solutions that connected method of wire and using a tensional object for reducing rotation. For proposed algorithm verification, we realized three axis motion control camera based on wire and measured oscillation while moving same trace. We confirmed the results that standard deviation of oscillation was reduced 4.93 degree than before design method.
\end{abstract}

Key Words : Motion control camera, anti-sway, oscillation, roll motion, yaw motion, motion control

\section{Introduction}

Recently, there is getting popular film using motion control camera in all over the world. Motion control camera is said that system by computer control for shooting same repetition [1]. Basically, this system has a track so that it can control elaborately horizontal movement and make complex motion of camera using variable axis (dolly, pan/tilt, roll, boom, swing, $\mathrm{x}$ track, focus and so on). It is moved motor which is controlled all axis by computer so that motion of camera is able to control precisely. Precise control of motion control camera can be obtained same camera view because of every shoot camera can move same by input data at each time. So, motion control camera is widely used various special effects shooting and broadcasting. Example, it is made thousands of people by multiplying images only using a little extra or it is made CG background using sequential shooting data for real scene [2, 3].

Now, motion control camera is widely used consist of track and boom, L-Head, camera. But it is enormous and occupied much space so that have been restricted space. Its price is also very expensive. Therefore, we proposed simple design method that is 3-axis motion control camera based on wires. It is able to use various spaces and can be made cheaper than original. Then, we expect to frequently use motion control camera than before and see great picture quality.

Proposed simple design method consists of rotary encoder and motor, wire, plate, camera. Rotary encoder measured wire length and motor controlled wire length then camera which is attached under plate is moved. We used straight-line move method to control precise position of plate. But when plate is

Manuscript received Nov. 17, 2009; revised Dec. 20, 2009.

* Corresponding author

This work was supported for two years by Pusan National University Research Grant. moved, it was occurred roll motion (roll axis lies along direction of camera angle) and we also suggested solution of this problem. The solution is that wires converge into one point. Although we solved the problem, another problem was occurred yaw motion. This problem solved to use a tensional object that caused energy distribution. We made proposed motion control camera system and experiment. And experiment result is that rotation of plate is reduced and camera moves to desired point.

The main objective of this paper is to develop the motion control camera system based on wires. With regard to proposed motion system, motion stability relied on anti-sway method to minimize roll and yaw motion. The following section provides a structure of proposed motion control system. Section 3 presents anti-sway methods to make stable movement. Section 4 and 5 shows motion control program and experimental result, and contains concluding remarks.

\section{Proposed System Design}

\subsection{Kinematic of Motion Control Camera Based on Wires}

We proposed the motion control camera system based on wires as shown in Fig. 1. (a). It is very simple structure and low-cost system. The system consists of three pipes and rotary encoders, motors, wires, and a plate, camera. We appeared 3axis by setting up pipes above edge of wall and both surfaces of a wall near ceil as shown in Fig. 1. (b). Also, we established three pulleys for controlling wires and rotary encoders for measuring wire length. Then wires length is changed using three motors in each axis so that a plate and camera under plate is controlled and moved by difference of three wires length. Therefore, we can appear hardware of motion control camera system based on wire. Next, we introduce various motion control method and our method [4-6]. 


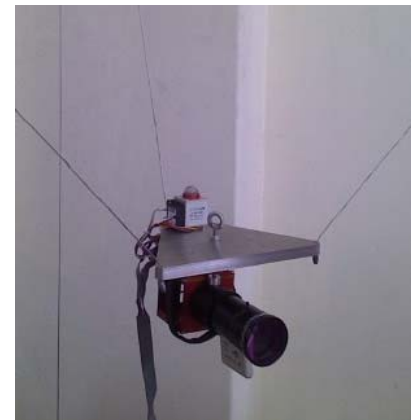

(a) Actual feature

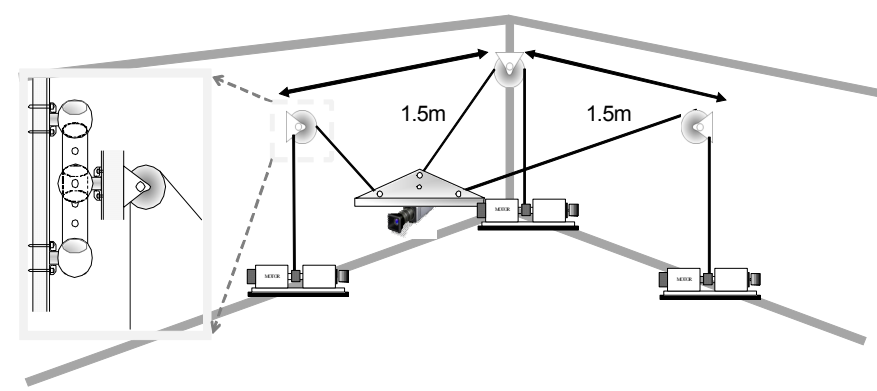

(b) Outline of motion control camera

Fig. 1 Proposed design of motion control camera.

\subsection{Motion Control Method}

There is three ways of motion control method which is straight-line move, arc move, and contoured move. A straightline move executes the shortest move between two points. Shortly, this method finds straight line of between two points. And arc move causes a coordinate space of axes to move on a circular, spherical, or helical path. We can move twodimensional vector spaces in a circle only on a $2 \mathrm{D}$ plane and move a 3D vector space on a spherical or helical path. Finally, a contoured move moves an axis or a coordinate space of axes in a pattern that we define. The trajectory generator on the motion controller is not used during a contoured move. The controller takes position data in the form of an array and splines the data before outputting it to the DACs or stepper outputs.

Because we regard that motion control camera is important to reaches repeatedly precise position, we selected straight-line move method. This method is simple and can easy use. First, we previously selected several objective points and calculated distance every two points. Then we can control the camera position by using calculated distance and motor. Calculation method is introduced next section [7-12].

\subsection{Position Control of Motion Control Camera}

We used straight-line method based position, so we need to calculate present position of motion control camera and motor velocity for controlling objective positions. Fig. 2. indicates simple figure of system for knowing variable at each point. This paper assumed that we know position variable of pulleys $T_{1}, T_{2}, T_{3}$ and initial wires length $L_{1}, L_{2}, L_{3}$.

We can calculate present camera position $P(x, y, z)$ using wires length $L_{1}, L_{2}, L_{3}$ is :

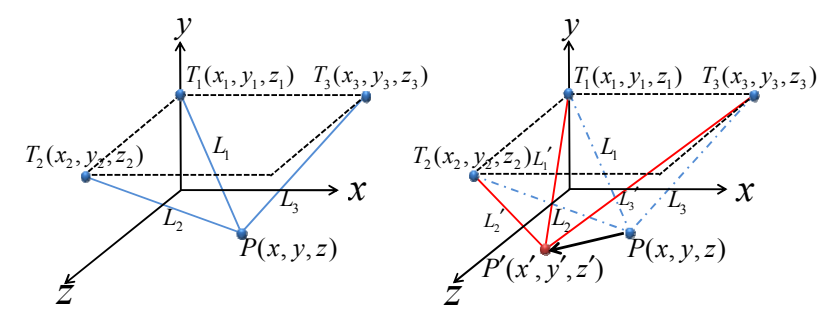

Fig. 2 Simple system positions by movement.

$$
\begin{aligned}
& \sqrt{\left(x-x_{1}\right)^{2}+\left(y-y_{1}\right)^{2}+\left(z-z_{1}\right)^{2}}=L_{1} \\
& \sqrt{\left(x-x_{2}\right)^{2}+\left(y-y_{2}\right)^{2}+\left(z-z_{2}\right)^{2}}=L_{2} \\
& \sqrt{\left(x-x_{3}\right)^{2}+\left(y-y_{3}\right)^{2}+\left(z-z_{3}\right)^{2}}=L_{3}
\end{aligned}
$$

Each polynomial expression (in (1) (3)) is calculated square and subtraction by following (4). Then, we obtained the present position of motion control camera.

$$
\begin{aligned}
(1)^{2}-(2)^{2} & =2\left(x_{2}-x_{1}\right) x+2\left(y_{2}-y_{1}\right) y+2\left(z_{2}-z_{1}\right) z \\
& =c_{1}-c_{2} \\
(2)^{2}-(3)^{2} & =2\left(x_{3}-x_{2}\right) x+2\left(y_{3}-y_{2}\right) y+2\left(z_{3}-z_{2}\right) z \\
& =c_{2}-c_{3} \\
(3)^{2}-(1)^{2} & =2\left(x_{1}-x_{3}\right) x+2\left(y_{1}-y_{3}\right) y+2\left(z_{1}-z_{3}\right) z \\
& =c_{3}-c_{1}
\end{aligned}
$$

where $c_{1}=L_{1}^{2}-x_{1}^{2}-y_{1}^{2}-z_{1}^{2}, c_{2}=L_{2}^{2}-x_{2}^{2}-y_{2}^{2}$ $-z_{2}^{2}, c_{3}=L_{3}^{2}-x_{3}^{2}-y_{3}^{2}-z_{3}^{2}$ and is calculated using initial wires length and pulleys positions. Thus, we can know present and next step is motor velocity calculation for moving objective position.

We assumed an objective position is $P^{\prime}\left(x^{\prime}, y^{\prime}, z^{\prime}\right)$ and we know $T_{1}, T_{2}, T_{3}$ and $L_{1}, L_{2}, L_{3}$. Then, we can know $L_{1}{ }^{\prime}, L_{2}{ }^{\prime}, L_{3}{ }^{\prime}$ is next polynomial expression (in (6)) and we obtain to variation of wire length $\Delta L$ in (11).

$$
\begin{gathered}
\sqrt{\left(x^{\prime}-x_{1}\right)^{2}+\left(y^{\prime}-y_{1}\right)^{2}+\left(z^{\prime}-z_{1}\right)^{2}}=L_{1}^{\prime} \\
\sqrt{\left(x^{\prime}-x_{2}\right)^{2}+\left(y^{\prime}-y_{2}\right)^{2}+\left(z^{\prime}-z_{2}\right)^{2}}=L_{2}^{\prime} \\
\sqrt{\left(x^{\prime}-x_{3}\right)^{2}+\left(y^{\prime}-y_{3}\right)^{2}+\left(z^{\prime}-z_{3}\right)^{2}}=L_{3}^{\prime} \\
\Delta L_{1}=L_{1}-L_{1}^{\prime} \\
\Delta L_{2}=L_{2}-L_{2}{ }^{\prime} \\
\Delta L_{3}=L_{3}-L_{3}{ }^{\prime}
\end{gathered}
$$


We decided motors velocity by $\Delta \mathrm{L} 1, \Delta \mathrm{L} 2, \Delta \mathrm{L} 3$. And we used normalized value for arriving objective position at the same time. So we can control motors velocity using rotary encoders that measured instantaneous wires length variation.

$$
\begin{aligned}
& v_{1}=\frac{\Delta L_{1}}{\text { Norm }} \times \text { Max velocity } \\
& v_{2}=\frac{\Delta L_{2}}{\text { Norm }} \times \text { Max velocity } \\
& v_{3}=\frac{\Delta L_{3}}{\text { Norm }} \times \text { Max velocity }
\end{aligned}
$$

where norm is maximum value of $\Delta \mathrm{L} 1, \Delta \mathrm{L} 2, \Delta \mathrm{L} 3$.

Then we tested for moving motion control camera using proposed solution. But it is unlike expecting, the plate is occurred problem that is the roll motion while moving. We proposed to solve this problem in next section.

\section{Anti-sway System Design}

\subsection{Approach the Roll Motion Problem and Solution}

We considered force of plate and wire for analyzing the roll motion. And this paper will be limited to consideration of 2axis about kinematic of plate and wires in static state as shown in Fig. 3.

The tensile force is resolved $\vec{F}_{T}$ and $\vec{F}_{N} \cdot \vec{F}_{T}$ which is defined according to (9). $\theta$ denotes the degree between plate and wire. $\vec{F}_{T_{1}}, \vec{F}_{T_{2}}$ are that the direction is opposite and the magnitude is equal in static state. Then we can calculate relation between $\vec{T}_{1}$ and $\vec{T}_{2}$.

$$
\begin{aligned}
& \vec{F}_{T}=\vec{T} \sin \theta \\
& \vec{F}_{T_{1}}=\vec{F}_{T_{2}} \\
& \vec{T}_{1} \sin \theta_{1}=\vec{T}_{2} \sin \theta_{2} \\
& \vec{T}_{1}=\frac{\sin \theta_{2}}{\sin \theta_{1}} \vec{T}_{2}
\end{aligned}
$$

Like (9), $\vec{T}_{1}$ and $\vec{T}_{2}$ depend on angle of between two wires and vertical direction of plate. When $\theta_{1}$ and $\theta_{2}$ differ, the tension forces have different value. Different tension forces cause the torque as shown (10) and the torque generated roll motion.

$$
\begin{aligned}
\tau & =\sum \vec{r} \times \vec{F}_{N}=\vec{r} \times \vec{F}_{N_{1}}+\vec{r} \times \vec{F}_{N_{2}} \\
& =\vec{r} \times \vec{T}_{1} \cos \theta_{1}+\vec{r} \times \vec{T}_{2} \cos \theta_{2}
\end{aligned}
$$

where $\tau$ is the torque, $r$ denotes the distance of between the center point of plate and a point of linking wire. Because roll motion caused eventually different $\theta_{1}, \theta_{2}$, we proposed to reduce the distance $r$ as shown in Fig. 4. If the distance $r$ is eliminated, it could be reduce the torque in (11). Therefore we can solve the problem of roll motion and we experimented to confirm but it is occurred to another problem which is Yaw motion. We also analyzed and proposed to solve this problem in next section.

$$
\begin{aligned}
\tau & =\sum \vec{r} \times \vec{F}_{N}=\vec{r} \times \vec{F}_{N_{1}}+\vec{r} \times \vec{F}_{N_{2}} \\
& =\vec{r} \times \vec{T}_{1} \cos \theta_{1}+\vec{r} \times \vec{T}_{2} \cos \theta_{2} \\
& =0 \times \vec{T}_{1} \cos \theta_{1}+0 \times \vec{T}_{2} \cos \theta_{2} \\
& =0
\end{aligned}
$$

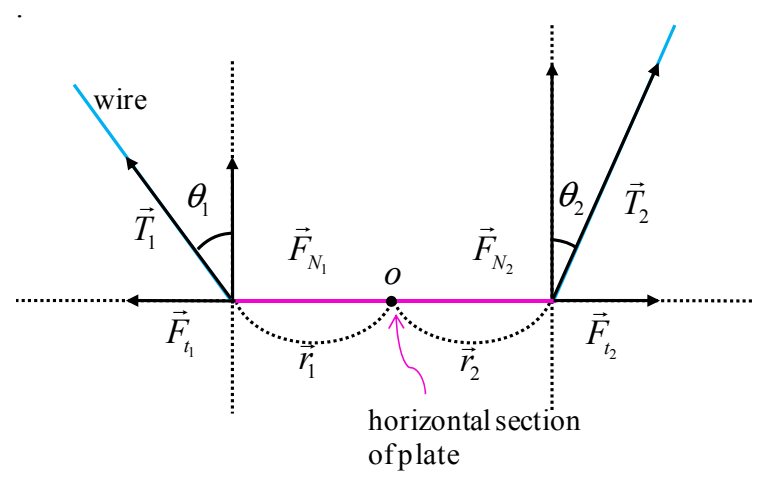

Fig. 32 axis to wires and plate of motion control camera.
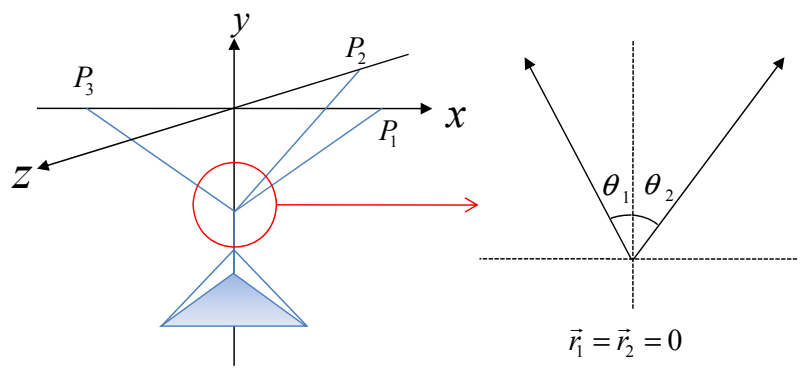

Fig. 4 Design to reduce problem of roll motion.

\subsection{Approach the Yaw Motion Problem and Solution}

To solve the yaw motion problem, we analyzed the vertex's force of plate as shown in Fig. 5 and the vertex's force expressed in (12).

$$
\begin{aligned}
& \vec{F}=\vec{T}_{1}+\vec{T}_{2}+\vec{T}_{3}=M \vec{g} \quad \text { (static-state) } \\
& \Delta \vec{F}=\sum \Delta\left(\vec{F}_{x}+\vec{F}_{z}\right)
\end{aligned}
$$

$\vec{F}_{y}$ indicates $M \vec{g}$ so that $\vec{F}_{y}$ eliminated and remained the force of $x, z$ directions. The torque related on the yaw motion is generated in (13).

$$
\tau=r \times \Delta \vec{F}_{(x+z)}
$$

We connected tensional objects between each wire and the vertex of plate for solving yaw motion as shown in Fig. 6. In Fig. 6., dotted line is tensional objects. We can prove proposed design to reduced yaw motion as shown in Fig. 7. This figure appeared the change in length of a tensional objects $l$ when it is occurred yaw motion and we found that the change of $l_{0}, l_{1}, l_{2}$ are similar to pendulum motion. In case of the pendulum motion, the position of $p_{0}$ has zero potential energy and 
maximum kinetic energy. In general, the energy of pendulum motion is defined by kinetic and potential energy in (14).

$$
E=\frac{1}{2} m v^{2}+m g h
$$

where $m$ denotes the mass, $v$ means the velocity, $g$ is the gravity acceleration, and $h$ is the height.

In proposed design of Fig. 6, the kinetic energy changes into strain and potential energy in pendulum motion. This is added strain energy differently before connected to tensional objects. Conversely, the potential energy changes into strain and kinetic energy in (15). As a result, kinematic and potential energy reduced due to strain energy.

$$
\begin{aligned}
& \frac{1}{2} m \Delta v^{2}=m g \Delta h+\frac{1}{2} k \Delta l^{2} \\
& m g \Delta h=\frac{1}{2} m v^{2}+\frac{1}{2} k \Delta l^{2}
\end{aligned}
$$

The energy relation of each position is defined according to:

$$
\begin{aligned}
& l_{0}: E_{0}=\frac{1}{2} m v_{0}^{2}+\frac{1}{2} k l_{0}^{2} \\
& l_{1}: E_{1}=\frac{1}{2} m v_{1}^{2}+\frac{1}{2} k l_{1}^{2}+m g h_{1} \\
& l_{2}: E_{2}=\frac{1}{2} m v_{2}^{2}+\frac{1}{2} k l_{2}^{2}+m g h_{2}
\end{aligned}
$$

$l_{1}$ and $l_{2}$ have strain energy and we know the principle of the conservation of energy. Then kinetic and potential energy of $l_{1}$, $l_{2}$ is reduced before. Therefore we confirmed to reduce yaw motion because of connecting tensional object's strain energy.

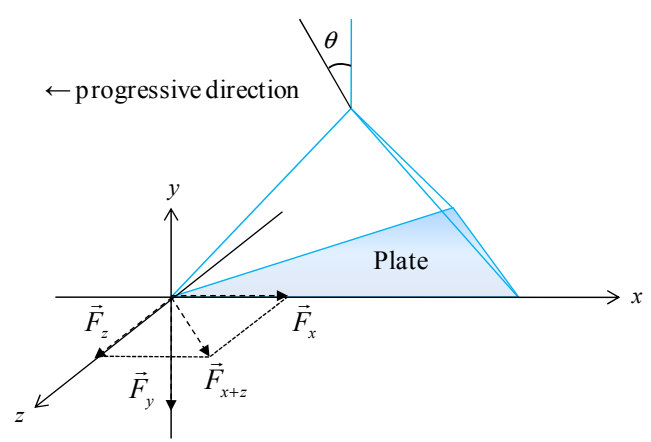

Fig. 5 The force relations of proposed design.
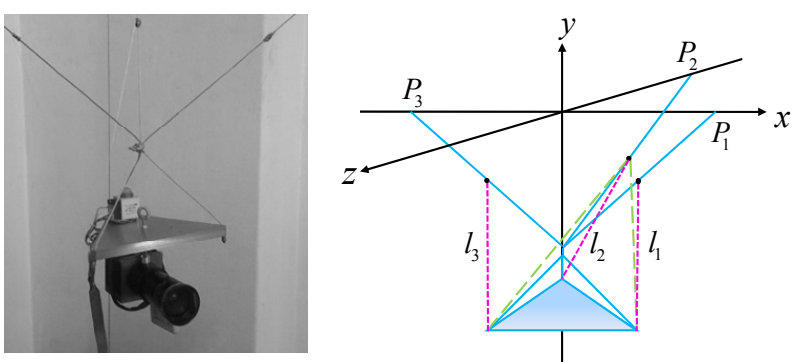

Fig. 6 The improved design using tensional object.

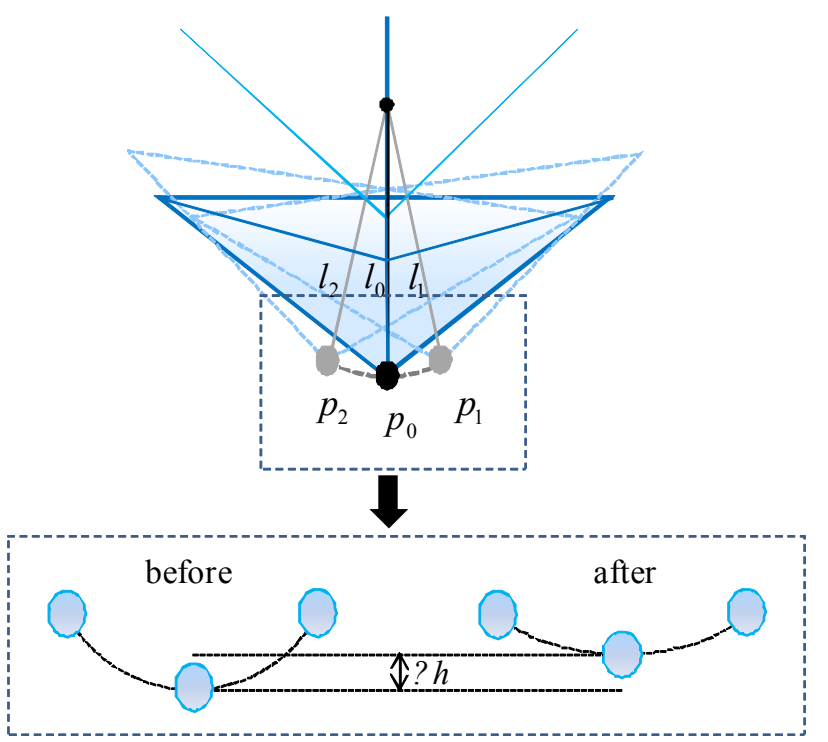

Fig. 7 The problem of pendulum motion of proposed design.

\section{Experimental Results}

We set up 3-axis motion control camera base on wire and we tested to verify proposed methods. The system needs a motion controller to operate three motors. The motion controller is PCI-7350 in National Instrument Corporation and used three motors are AC servo motor, FMA CK04-AB00 in HIGEN Corporation. And we used rotary encoder for measuring wire length. Two tilt sensors (DAS-M1) measured roll and pitch rotation of the plate. All of hardware is controlled development software LABVIEW 8.6. To experiment, we appointed several points and the system is moved as selected order of points. In Fig. 8. indicates our experiment method for tilt measurement. Right figure of Fig. 8. is shown motion control program using LABVIEW. It has monitor mode and manual mode, auto mode. Monitor mode is able to check present position and wire length, motor velocity and so on. And manual mode is passive moving and auto mode is used automatic action that is previously gave trajectory. Using auto mode of developed system, we selected trajectory followed arrow like ' 8 ' with starting point $(0,0)$. We measured roll and pitch axis tilts of plate during moving camera for comparing with anti-sway design and without antisway. Shortly, we measured roll tilts before and after improving roll motion. Likewise, we also measured pitch tilts. Experimental result is shown that proposed anti-sway design is better than without anti-sway design. We described the variance of tilt and compared with roll and pitch direction in Fig. 9. And we expressed the Gaussian distribution of rotation on each axis in Fig. 10. We calculated the standard deviation from Gaussian distribution. In roll axis, without anti-sway design has 1.8567 and anti-sway design has 1.1085. And without anti-sway design has 6.3480 and anti-sway design has 1.4147 in pitch axis tilt. Therefore we are shown that rotation of plate is reduced both roll and pitch axis and concluded that performance of proposed design is good. 

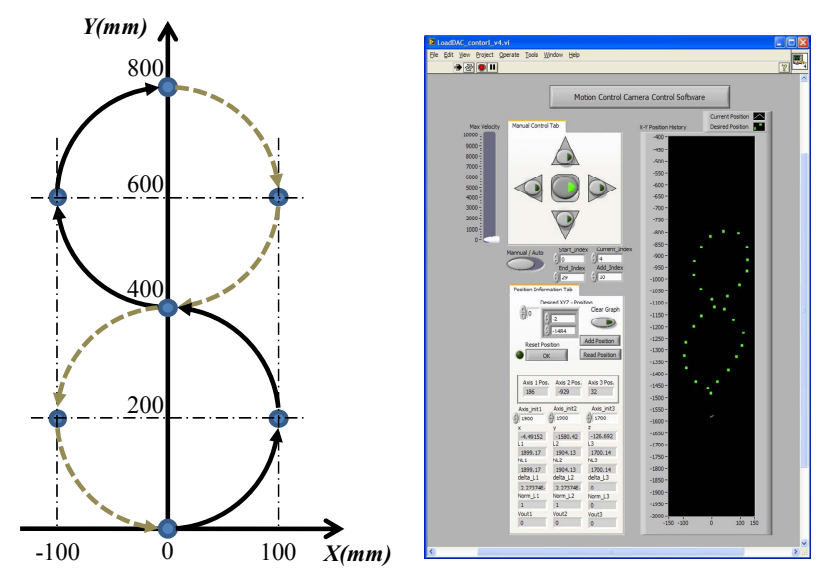

Fig. 8 The experimental trajectory and motion control program.

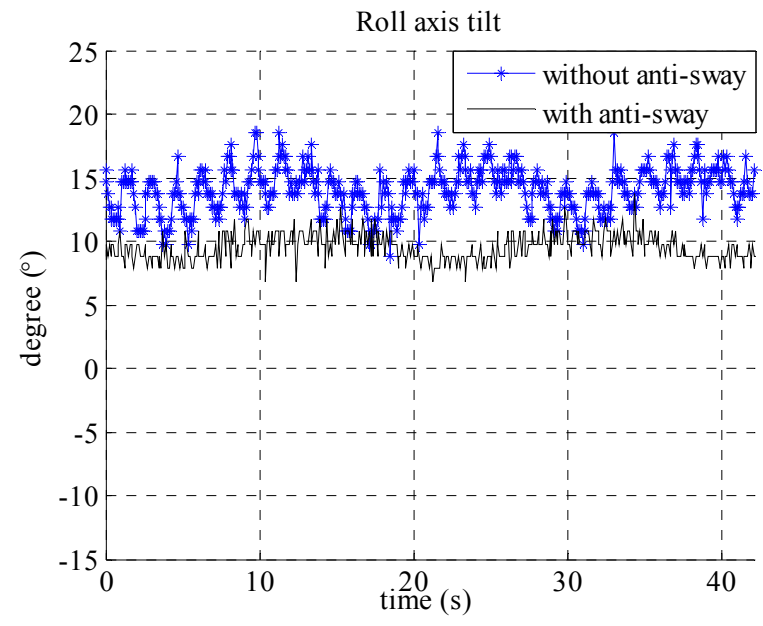

(a) Roll axis tilt of first design and advanced design

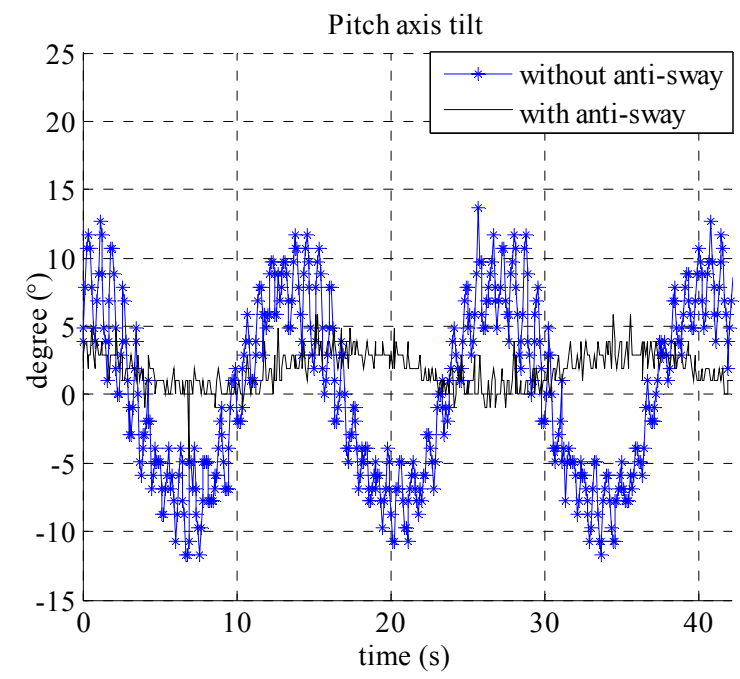

(b) Pitch axis tilt of first design and advanced design

Fig. 9 Roll and Pitch axis tilt value.

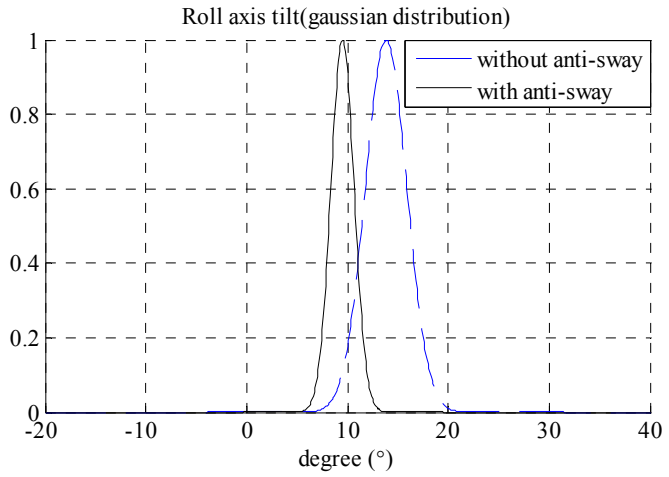

(a) Roll axis tilt of first design and advanced design

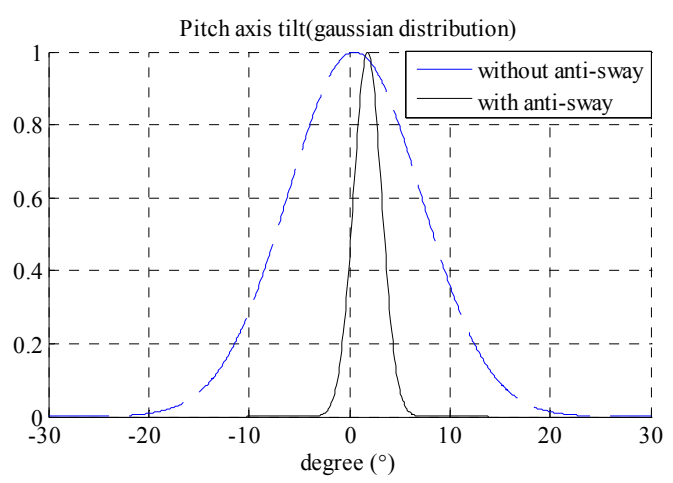

(b) Pitch axis tilt of first design and advanced design

Fig. 10 Gaussian distribution of Roll, Pitch axis tilt.

\section{Conclusion}

This paper proposed 3-axis motion control camera design based on wires and moving control algorithm used straight-line move method because we wanted to move repeatedly precise position. But system had some problems that roll and yaw motion. So we approached and solved the problem. The problem of roll motion is solved with eliminating the distance of between the center point of plate and a point linking wire. And the problem of yaw motion is solved with connecting tensional objects. We tested design that has each problem and applied anti-sway design. As a result, we confirmed proposed anti-sway design is advanced.

Proposed motion control camera design based on wires is simple structure and cheaper so that is able to use various place. But proposed system is hard to smooth trajectory control in curve path, because the control algorithm of proposed system used position based straight line of straight-line move method. So there remains to be more studied the motion attitude and when we have a chance, we will apply control algorithm that is arc move and contoured move. If control algorithm is developed the dynamic moving method, proposed system will be widely available motion control camera to handle image processing. 


\section{References}

[1] Jody Duncan, "The Making of The lost World : Jurassic Park," Ballantine Books, New York, May 1997.

[2] Hiroyasu Masuda, Akira Akahoshi, Tetsuaki Nakazawa, "Development of a Compact Motion Control Camera System for HD Digital Broadcasting," SMPTE Technical Conference and Exhibition, New York, pp. 1-10, Nov. 2005.

[3] Lee Won-jae, Lee Sung-sik, "A Case Study on Special Effect of Object Animation through Motion Control Camera," Korean Society of Design Science, vol. 46, pp. 164-165, May 2002.

[4] Bernardo Uribe Mendoza, Luis Miguel Mendez, "Camera Motion Control from a Java 3D Environment : Virtual Studio Application in Decorative Arts Museum Collections," Proceedings of the 2006 International Conference on Cyberworlds, pp. 58-64, Nov. 2006.

[5] Richard Y.D.Xu, Joshua M. Brown, Jason M. Traish, Daniel Dezwa, "A Computer Vision Based Camera Pedestal's Vertical Motion Control," International Conference on Pattern Recognition (ICPR 2008), pp. 1-4, Dec. 2008.

[6] Frederic Benhamou, Frederic Goualard, Eric Languenou, Marc Christie, "Interval Constraint Solving for Camera Control and Motion Planning," ACM Transactions on Computational Logic, vol. 5, no. 4, pp. 732-767, Oct. 2004.

[7] Han Ding, Jianjua Wu, "Point-to-Point Motion Control For a High-Acceleration Positioning Table via Cascaded Learning Schemes," IEEE Trans. on Industrial Electronics, vol. 54, no.5, pp. 2735-2744, Oct. 2007.

[8] Arun D. Mahindrakar, Shodhan Rao, R.N.Banavar, "Point-topoint control of a $2 \mathrm{R}$ planar horizontal underactuated manipulator," Mechanism and Machine Theory, vol. 41, no. 7, pp. 838-844, Jul. 2006.

[9] Zhi Xhou, Kok-Meng Lee, "Real-time Motion Control of a Multi-degree-of-freedom Variable Reluctance Spherical Motor," Proceedings of the 1996 IEEE International Conference on Robotics and Automation, vol. 3, pp. 2859-2864, Apr. 1996.

[10] Robert D. Lorenz, Thomas A. Lipo, Donald W. Novotny, "Motion Control with Induction Motors," Proceedings of the IEEE, vol. 82, no. 8, pp. 1215-1240, Aug. 1994

[11] Dong Sun, James K. Mills, "Torque and Current Control of High-Speed Motion Control Systems With Sinusoidal-PMAC Motors," IEEE/ASME Trans. on Mechatronics, vol. 7, no. 3, pp. 369-377, Sep. 2002

[12] W. Steiger, N. Sherkat, P.D. Thomas, "A Stepping Motor Control Algorithm for Smooth Continuous Path Motion," International Conference on Control, vol. 1, pp. 816-821, 1994

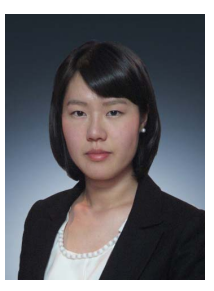

Taerim Kim

She received the B.S. degree in the school of electronic, electrical and communication engineering from Pusan National University, Busan, Korea, and is currently working in the M.S. course. Her research interests include intelligent control, fuzzy logic control, and robot.

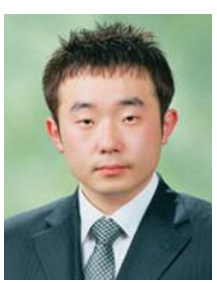

\section{Sungyoung Jung}

He received the B.S. degree in the school of electronic, electrical and communication engineering from Pusan National University, Busan, Korea, and is currently working in the M.S. course. His research interests include intelligent control, pattern recognition and robot.

\section{Gyeongdong Baek}

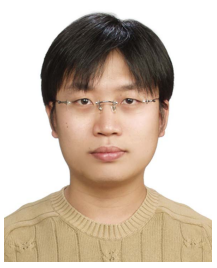

He received the B.S and M.S. degrees in electrical engineering from Pusan National University, Busan, Korea, in 2008. He is currently pursuing the $\mathrm{Ph}$. D. degree at Pusan National University. His research interests include intelligent control and fault diagnosis.

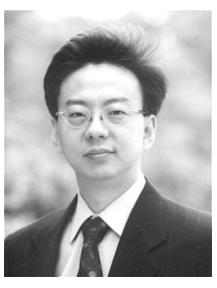

\section{Sungshin Kim}

$\mathrm{He}$ received the B.S. and M.S. degrees in electrical engineering from Yonsei University, Seoul, Korea, in 1984 and 1986, respectively, and the Ph.D. degree in electrical and computer engineering from Georgia Institute of Technology, Atlanta, in 1996. He is currently Professor in the School of Electrical Engineering, Pusan National University, Busan, Korea. His research interests include intelligent control, fuzzy logic control, hierarchical learning structures, manufacturing systems, and data mining.

$\begin{array}{ll}\text { Phone } & :+82-51-510-2374 \\ \text { Fax } & :+82-51-513-0212 \\ \text { E-mail } & : \text { sskim@pusan.ac.kr }\end{array}$

